\title{
Introducing an Efficient In Vitro Cornea Mimetic Model for Testing Drug Permeability
}

\author{
Agnè Žiniauskaitè 1,4, Vytautas Cèpla 2,3, Tadas Jelinskas ${ }^{3}$, Romuald Eimont 2,3, Artūras Ulčinas ${ }^{3}$, Rūta Aldonytè 2,4 \\ Ramūnas Valiokas 2,3, Giedrius Kalesnykas 1,5 and Jenni J. Hakkarainen ${ }^{5, *}$ \\ 1 UAB Experimentica, Saulètekio ave 7C, LT-10223 Vilnius, Lithuania \\ 2 UAB Ferentis, Savanoriu 231, LT-02300, Vilnius, Lithuania \\ 3 Department of Nanoengineering, Center for Physical Sciences and Technology, Savanoriu 231, LT-02300, \\ Vilnius, Lithuania \\ 4 State Research Institute Center for Innovative Medicine, Santariskiu 5, Vilnius, Lithuania \\ 5 Experimentica Ltd., Microkatu 1, P.O. Box 1199, FI-70211 Kuopio, Finland \\ * Correspondence: Jenni J. Hakkarainen (jenni@experimentica.com)
}

\begin{abstract}
There is a growing need for novel in vitro corneal models to replace animal-based ex vivo test in drug permeability studies. In this study we demonstrate a corneal mimetic that models the stromal and epithelial compartments of human cornea. Human corneal epithelial cells (HCE-T) were grown on top of a self-supporting porcine collagen-based hydrogel. Cross sections of the multi-layers were characterized by histological staining and immunocytochemistry of zonula occludens-1 protein (ZO-1) and occludin. Furthermore, water content and elastic properties of the synthetized collagen type I-based hydrogels were measured. The apparent permeability coefficient ( $\mathrm{Papp}$ ) values of a representative set of ophthalmic drugs were measured and correlated to rabbit cornea Papp values found in the literature. Multilayered structure of HCE-T cells and expression of ZO-1 and occludin in full thickness of multilayer were observed. The hydrogel-based corneal model exhibited excellent correlation to rabbit corneal permeability $(\mathrm{r}=0.96)$, whereas insert-grown HCE-T multilayer was more permeable and the correlation to the rabbit corneal permeability was lower $(\mathrm{r}=0.89)$. The hydrogel-based human corneal model predicts the rabbit corneal permeability more reliably in comparison to HCE-T cells grown in inserts. This in vitro human corneal model can be successfully employed for drug permeability tests whilst avoiding ethical issues and reducing costs.
\end{abstract}

Keywords: in vitro corneal model; collagen hydrogel; permeability

\section{Introduction}

Topical administration of ophthalmic drugs remains the preferred route of drug delivery to ocular tissues. However, poor bioavailability of drugs often limits their efficacy. The cornea decreases drug bioavailability due to its complex layered structure comprising of stratified epithelium, Bowman's layer, stroma, Descemet's membrane and, finally, the endothelial layer. The corneal epithelium itself is composed of layered squamous, wing and basal cells. The superficial apical layer of the epithelial cells contains tight junctions that seal the intercellular spaces between them making the corneal epithelium into an efficient diffusion barrier. Therefore, the corneal epithelium represents a rate-limiting barrier for topically administered hydrophilic drugs and macromolecules. Corneal stroma consists mostly of water ( 80\%) and type I collagen fibrils and makes up to $90 \%$ of the thickness of the cornea $[1,2]$. Therefore, with these properties, the corneal stroma restricts the penetration of hydrophobic drug molecules effectively [1].

Directive 2010/63/EU of the European Parliament and the Council on the protection of animals used for scientific purposes strongly supports the development and validation of non-animal methods and models [3]. Therefore, the ethical issues have become an 
important aspect for society, authorities and scientists. However, excised animal corneas (ex vivo) are still being used for permeability testing [4-6]. There are many disadvantages in use of animal ex vivo corneas, including high numbers of animals being sacrificed and, subsequently, high costs involved. Thus, innovative and validated in vitro methods are needed to replace the current ex vivo methods.

Three-dimensional (3D) corneal tissue models have been studied and described before $[7,8]$. These in vitro models offer a faster, cheaper, and ethically more appropriate tools for drug development. However, the permeability data of clinically used ophthalmic drugs through these models is insufficient. To the best of our knowledge, a very few $3 \mathrm{D}$ models of human cornea with permeability data sets of a wide range of compounds have been published, however the permeability properties of previously established models deviated from permeability of rabbit corneas [9-11]. Moreover, cell-based corneal models are still at the early stages of development. Nevertheless, their promising potential for the use in pharmacokinetic, penetration and toxicity studies are driving research forward. Several types of cells have been used for in vitro cornea modeling, for example, epithelial cells [12-14] as well as epithelial cells cultured together with stromal cells [8-11] The supporting matrix in corneal models is often collagen-based as it mimics the chemistry of the native corneal stroma where the collagen fibrils are enriched with proteoglycans $[15,16]$.

There has been an extensive research and development on the already established corneal in vitro models mentioned above. Nevertheless, the major drawback in most of the studies comparing in vitro data with excised animal corneas is the use of different permeability apparatuses resulting in substantial differences in permeability coefficients [17]. Therefore, the aim of this study was to develop a self-supporting, stable and transparent hydrogel platform. To reliably compare the permeability coefficients, such a testing platform would employ the regular permeability apparatus commonly used for most of the excised rabbit corneal permeation studies.

The technical challenge in developing disposable in vitro constructs compatible with the standard drug permeability instrumentation, is the synthesis of stable hydrogel material without compromising its permeability and cell-supporting characteristics. Moreover, beside the (bio)chemical composition and the characteristic fibrous ultrastructure, the elasto-mechanic properties of the hydrogel must match those of the native corneal tissue, triggering the mechanobiological cues that facilitate efficient cellular multilayering [18]. Therefore, in this paper, we present an in vitro corneal mimetic where a chemically crosslinked collagen-based self-supporting hydrogel is employed as a matrix for human corneal epithelial multilayer. We have performed quantitative assessment of the permeability of pharmacologically relevant ophthalmic drugs and compared it with the current in vitro and ex vivo standards, human corneal epithelial multilayer grown in cell culture inserts and rabbit cornea, respectively. A high correlation in permeability properties of our corneal mimetic was achieved with the rabbit cornea enabling further translation of this in vitro system into the pharmaceutical field and successful replacement of conventionally used excised rabbit tissues.

\section{Materials and Methods}

\subsection{Synthesis and Characterization of Hydrogels}

Collagen hydrogel membranes were synthesized from chemically crosslinked porcine collagen type I (NMP collagen PS, Nippon Meatpackers, Ibaraki, Japan) adapting the published protocol [19]. Briefly, collagen at $12 \%(\mathrm{w} / \mathrm{w})$ in aqueous solution was crosslinked with 4-(4,6-dimethoxy-1,3,5-triazin-2-yl)-4-methylmorpholinium chloride (DMTMM, Merck KGaA, Darmstadt, Germany) [20]. The amine molar ratio of collagen$\mathrm{NH} 2$ to DMTMM was 1:1. After thoroughly mixing, the final solution containing $8.5 \pm 0.3$ $\%$ collagen was casted onto a plastic circular supporting frame made from poly-L-lactic acid (PLLA), provided by Ferentis (Vilnius, Lithuania). The external diameter of the frame was $19.6 \pm 0.6 \mathrm{~mm}$, the inner diameter (corresponding to the diameter of the hydrogel membrane) was $10.8 \pm 0.4 \mathrm{~mm}$ and the thickness was $0.8 \pm 0.1 \mathrm{~mm}$. The obtained hydrogel membranes in the frames were left to cure overnight in a 100\% humid atmosphere. After 
curing, the hydrogel membranes were washed several times with $0.1 \mathrm{M}$ phosphate buffer (PB) (pH 7.4) and kept in 0.1 M PB (pH 7.4) supplemented with $100 \mathrm{U} / \mathrm{ml}$ penicillin, 100 $\mu \mathrm{g} / \mathrm{ml}$ streptomycin and $0.25 \mu \mathrm{g} / \mathrm{ml}$ Fungizone (Gibco, Thermo Fischer Scientific, Waltham, MA, USA) until use.

The elastic (Young) moduli $\mathrm{E}$ of the synthesized hydrogel membranes was determined by the atomic force microscopy (AFM) nanoindentation technique [21,22]. The spring constant of the tipless AFM cantilevers (NSC36-B, MicroMasch) was calibrated using the thermal noise method [23], before the attachment of a microsphere and was found to be $4.0 \pm 0.6 \mathrm{~N} / \mathrm{m}$. For hydrogel analysis, the probes from the same synthesis batch were chosen because of the observed low variation in the spring constant values. To control the geometry of the contact, silicon dioxide microspheres (Microparticles $\mathrm{GmbH}$, Berlin, Germany) with a manufacturer-reported diameter of $6.65 \pm 0.28 \mu \mathrm{m}$ were attached to the AFM cantilevers using UV-curable glue (NOA68, Norland), taking care to control the amount of the glue on the cantilever. The attachment of the microspheres and their diameters were inspected by optical microscopy (a 50× lens, BX51, Olympus, Japan). The measured diameter was $6.65 \pm 0.15 \mu \mathrm{m}$, matching the data provided by the manufacturer.

To avoid the movement of the sample during the AFM elastic modulus measurement, cutouts of the hydrogel membranes with a typical width of $3 \mathrm{~mm}$ and a length of 5 $\mathrm{mm}$ were attached to the (3-aminopropyl)trimethoxysilane-treated glass substrates (BaltFab, Vilnius, Lithuania) cut from regular carrier slides. The attachment was secured via glutaraldehyde chemistry. In a typical experiment, one glass substrate had two to five hydrogel pieces mounted. For elastic modulus determination hydrogel sample was placed in a small Petri dish filled with phosphate buffered saline (PBS, pH 7.4) and mounted onto the stage of an inverted optical microscope (IX73, Olympus, Japan), which served as a base for the AFM instrument (Nanowizard 3, JPK, Germany). The nanoindentation probe was approached to a bare glass surface and the detection sensitivity factor was determined from the force-displacement curve. The typical detection sensitivity values were 10-20 $\mathrm{nm} / \mathrm{V}$. The probe was then repositioned onto the hydrogel sample and 1024 force curves were collected from the $50 \times 50 \mu \mathrm{m}^{2}$ area in the Quantitative Imaging ${ }^{\mathrm{TM}}$ (QI, JPK) mode with a force setpoint of $120 \mathrm{nN}$ and a loading rate of $50 \mu \mathrm{m} / \mathrm{s}$. For each sample analyzed, this procedure was repeated at four different locations. The obtained curves were analyzed, and the E values of the hydrogel samples were extracted by fitting the approach part of the force-displacement curve with the Hertz sphere-on-plane elastic contact model using the JPK data processing software (version spm-5.0.84). The elastic moduli E were calculated from the analysis of three different synthesis batches of four hydrogel discs (12 samples in total).

\subsection{Cell Culture of Human Corneal Epithelial Cells}

The human corneal epithelial cell line (HCE-T) [24] was obtained from the RIKEN cell bank (Tsukuba, Japan). The HCE-T cells were grown in DMEM/F12 (1:1) media supplemented with $5 \%$ fetal bovine serum, $100 \mathrm{U} / \mathrm{ml}$ penicillin - $100 \mu \mathrm{g} / \mathrm{ml}$ streptomycin, 5 $\mu \mathrm{g} / \mathrm{ml}$ insulin, $10 \mathrm{ng} / \mathrm{ml}$ human recombinant epidermal growth factor (all from Gibco, Thermo Fischer Scientific) and 0.5\% dimethyl sulfoxide (Sigma-Aldrich, St. Louis, MO, USA) in an incubator, with a humidified atmosphere of $5 \% \mathrm{CO}_{2}$ and a temperature of $37^{\circ} \mathrm{C}$ [25]. The cell suspension was applied on the top of the collagen hydrogel in their supporting frames and placed into 6-well cell culture plates (Cellstar ${ }^{\circledR}$, Greiner Bio-One, Kremsmünster, Austria). The seeding density was $50000 \mathrm{cells} / \mathrm{cm}^{2}$ and the cells were allowed to attach for 20 minutes prior to filling the wells with media. Cultures were maintained in $5 \% \mathrm{CO}_{2}$ at $37^{\circ} \mathrm{C}$ for one week. Thereafter, the HCE-T layer was cultured at the air-liquid interface by lifting the collagen hydrogels, with their supporting frames, onto permeable cell culture inserts with a diameter of $30 \mathrm{~mm}$ (Millicell, Sigma-Aldrich). Culture medium was added at the basolateral side of the insert membrane, changed three times a week, and the cultures were further maintained for two weeks. Control HCE-T cultures were seeded directly onto permeable $\left(0.4 \mu \mathrm{m}\right.$ pores) cell culture inserts (Corning ${ }^{\circledR} \operatorname{Costar}^{\circledR}$ 
Snapwell, $12 \mathrm{~mm}$, Corning, NY, USA), cultured until confluence for one week and then stratified for three weeks at the air-liquid interface as described previously [26].

\subsection{Permeability Testing}

Cytotoxicity of the drug molecules, at selected test concentrations (Table 1), was assessed using resazurin cell viability assay as previously described [25] with no signs of cytotoxicity (data not shown).

Table 1. Ophthalmic drugs and reference molecules tested in the permeability experiments.

\begin{tabular}{ccc}
\hline Ophthalmic drug / Reference molecule & Supplier & Concentration tested \\
\hline 6-carboxyfluorescein (6-CF) & Sigma-Aldrich & $50 \mu \mathrm{M}$ \\
Rhodamine B (Rho B) & Sigma-Aldrich & $50 \mu \mathrm{M}$ \\
FITC-dextran, 4 kDa (FD4) & Sigma-Aldrich & $50 \mu \mathrm{M}$ \\
FITC-dextran, 70 kDa (FD70) & Sigma-Aldrich & $200 \mu \mathrm{g} / \mathrm{ml}$ \\
Rhodamine 123 (Rho 123) & Sigma-Aldrich & $10 \mu \mathrm{M}$ \\
Betaxolol (Beta) & Cayman Chemicals & $10 \mu \mathrm{M}$ \\
(+)-Pilocarpine HCl (Pilo) & Cayman Chemicals & $10 \mu \mathrm{M}$ \\
Timolol maleate (Timo) & Cayman Chemicals & $10 \mu \mathrm{M}$ \\
Chloramphenicol (Chlora) & BioChemica, AppliChem & $80 \mu \mathrm{M}$ \\
Dexamethasone (Dexa) & Cayman Chemicals & $100 \mu \mathrm{M}$ \\
Brinzolamide (Brinzo) & Cayman Chemicals & $89 \mu \mathrm{M}$ \\
\hline
\end{tabular}

The permeability experiments were conducted in a NaviCyte vertical chamber system (Harvard Apparatus, Holliston, MA, USA). Briefly, the cell layers or rabbit corneas with scleral rim were washed, equilibrated in pre-warmed BSS Plus buffer (Alcon, Fort Worth, Texas, USA) for 15 minutes, and then placed between the donor and receiver chambers. The donor chamber was filled with $5.5 \mathrm{ml}$ donor solution and the receiver chamber was filled with $6.5 \mathrm{ml}$ of BSS Plus to mimic the intraocular pressure in the receiver chamber. Solutions in chambers were mixed individually using carbogen $(95 \%$ oxygen and $5 \%$ carbon dioxide) gas. The samples were collected from the receiver chambers at $10,15,30,45,60,90$, and $120 \mathrm{~min}$ and the samples from the donor chambers were collected at 30, 60, 90, and $120 \mathrm{~min}$ for in vitro corneal models. The samples were collected from the receiver chambers at 30,60,120, 150, 180, 210, and $240 \mathrm{~min}$ and the samples from the donor chambers were collected at $0,60,180$, and $240 \mathrm{~min}$ for the rabbit corneas. The sample concentrations of the fluorescent molecules (FD4, FD70, Rho 123, Rho B and 6-CF) were quantified in the 96-well plates using a Cytation 3 multi-mode reader (BioTek Instruments, Winooski, VT, USA). A minimum of five cell inserts were used to test the permeability of clinically used ophthalmic drugs and fluorescent reference molecules listed in the Table 1. The permeability of fluorescent reference molecules (FITC-dextran $4 \mathrm{kDa}$, 6-carboxyfluorescein, Rhodamine 123 and Rhodamine B) were tested across rabbit corneas, a minimum of four corneas were used per molecule. The results obtained for the corneal model with collagen hydrogel and for the cell layers grown in cell culture inserts were compared with the permeability results of rabbit corneas from existing published studies supplemented with data obtained in this study.

\subsection{Quantification of Brinzolamide, Dexamethasone, Chloramphenicol, Timolol, Pilocarpine and Betaxolol}

The samples collected from receiver and donor chambers in permeability tests were prepared for analysis by protein precipitation using one-fold dilution of acetonitrile, followed by centrifugation for pellet compaction. Analytical ultra-performance liquid chromatography (UPLC) with MS/MS detection were used for quantification of model drug concentrations from the permeability test samples. The instrumentation consisted of Thermo Vanquish Horizon UPLC + Thermo Quantis triple quadrupole MS with HSS T3 column $(1.8 \mu \mathrm{m}, 2.1 \times 30 \mathrm{~mm})$ (Waters Corporation, Milford, MA, USA). For each analyte, separate nine-point calibration curves $(1-10000 \mathrm{nM})$ were prepared using the BSS Plus buffer. In addition to standards, the sequences included blank and zero samples and quality control samples (50, 500 and $5000 \mathrm{nM}$ ). For dexamethasone, chromatographic 
separation was preformed using gradient elution with (A) $2 \mathrm{mM}$ ammonium formate ( $\mathrm{pH}$ 8.5) and (B) acetonitrile. For brinzolamide, chloramphenicol, timolol, pilocarpine and betaxolol, chromatographic separations were preformed using gradient elution with (A) $2 \mathrm{mM}$ ammonium formate ( $\mathrm{pH}$ 8.5) and (B) methanol as follows: 0-0.5 min: $2 \% \mathrm{~B} ; 0.5-1.0$ $\min 2 \%$ B $\rightarrow 80 \%$ B; $1.0-1.75$ min: $80 \%$ B $\rightarrow 98 \%$ B; $1.75-2.25$ min: $98 \%$ B $\rightarrow 2 \%$ B. Flow rate was $0.65 \mathrm{ml} / \mathrm{min}$, column temperature $40^{\circ} \mathrm{C}$ and injection volume $4 \mu \mathrm{l}$. The following ionization conditions were used: nitrogen sheath, auxiliary and sweep gas flow rates 50, 4 and 4 instrument units, respectively and spray voltage $2 \mathrm{kV}$ (ESI+) and $3.5 \mathrm{kV}$ (ESI-). Analyte detection was performed using the following transitions: $\mathrm{m} / \mathrm{z} 393 \rightarrow 355$ for dexamethasone, $\mathrm{m} / \mathrm{z} 384 \rightarrow 136$ for brinzolamide, $\mathrm{m} / \mathrm{z} 321 \rightarrow 194$ for chloramphenicol, m/z 317 $\rightarrow 244$ for timolol, m/z $209 \rightarrow 121$ for pilocarpine and m/z $308 \rightarrow 121$ for betaxolol. For quantification, peak area ratios of the analyte and the internal standard were calculated as a function of the concentration of the analyte using Xcalibur 4.1 software (Thermo Scientific).

\subsection{Histology}

After the permeability tests, the cell layers were washed three times with BSS Plus and fixed in methanol (Fisher Scientific, Hampton, USA) at $-20^{\circ} \mathrm{C}$ for $20 \mathrm{~min}$. Rabbit cornea was fixed in $4 \%$ paraformaldehyde (Sigma-Aldrich) at $+4^{\circ} \mathrm{C}$ overnight. After fixation, the samples washed with PBS and embedded into optimal cutting temperature O.C.T. medium (Tissue Tek ${ }^{\circledR}$, Sakura Finetek USA, Torrance, CA, USA). The sections $(5 \mu \mathrm{m}, 10 \mu \mathrm{m}$ and $30 \mu \mathrm{m}$ for HCE-T cells grown in insert, rabbit cornea and HCE-T cells grown on top of hydrogel, respectively) were cut using a cryostat (Leica CM1860, Leica Biosystems, Wetzlar, Germany) and collected on SuperFrost ${ }^{\circledR}$ (Thermo Fisher Scientific) slides and stored at $-20^{\circ} \mathrm{C}$ until further use. Sections were stained with hematoxylin (Sigma-Aldrich) and eosin (Merck Millipore, Darmstadt, Germany). The stained sections were then visualized under a light microscope (Leica DLMB 100S, Leica Microsystems, Wetzlar, Germany) equipped with the LAS EZ software (Leica).

\subsection{Immunofluorescent Staining of Tight Junction Proteins}

Cryosections were washed with PBS, permeabilized, then blocked with 5\% normal goat serum (NGS) (Biowest, Nuaille, France) and 0.5\% Triton X-100 (Sigma-Aldrich) in PBS for $20 \mathrm{~min}$. The sections were then incubated at $+4^{\circ} \mathrm{C}$ overnight with rabbit anti-zonula occludens-1 protein (ZO-1) antibody (dilution 1:100, Thermo Fisher Scientific) and mouse anti-occludin antibody (1:200, Thermo Fisher Scientific). Mouse IgG isotype control (Abcam plc, Cambridge, UK) was used as negative control for rabbit tissue sections. After washing in $0.5 \%$ NGS in PBS, the sections were incubated with goat anti-rabbit secondary antibody, conjugated with Alexa Fluor 488 (1:500) or Alexa Fluor Plus 647 (1:500) or goat anti-mouse IgG1 secondary 488 (1:500) (Thermo Fischer Scientific), for 3h and washed in PBS. Finally, nuclei were stained with $0.1 \mu \mathrm{g} / \mathrm{ml}$ 4',6-Diamidino-2-phenylindole dihydrochloride (DAPI) (Sigma-Aldrich) for 30 minutes. The fluorescent images were acquired in an Axio Imager, on ApoTome.2 fluorescence microscope (Carl Zeiss, Oberkochen, Germany) and Leica THUNDER 3D Tissue Imager (Leica Microsystems).

\subsection{Data Analysis}

The apparent permeability coefficient $\left(\mathrm{P}_{\mathrm{app}}\right)$ value $(\mathrm{cm} / \mathrm{s})$, describing the rate at which molecule cross the barrier, was calculated using equation:

$$
P_{\text {app }}=\left(\Delta \mathrm{Q}_{\mathrm{r}} / \Delta \mathrm{t}\right)(1 /(\mathrm{AC} \mathrm{d})),
$$

where $\Delta \mathrm{Q}_{\mathrm{r}} / \Delta \mathrm{t}$ is a slope of a linear region of the cumulative amount of the molecule in the receiver chamber versus time, $A$, is the surface area available for diffusion, and $C_{d}$ is an average concentration of the molecule in the donor chamber.

The mass balance (\%) was calculated according to the following equation: 


$$
\text { Mass balance }(\%)=\frac{\left(C_{r(z)} * V_{r}\right)+\left(C_{d(z)} * V_{d}\right)}{\left(\text { average } C_{d} * V_{d}\right) * 100}
$$

where $C_{r}$ and $C_{d}$ are the concentrations in receiver and donor chambers, respectively, at the end $(z)$ of the permeability assay; $V_{r}$ and $V_{d}$ are the volumes of the receiver and donor chambers, respectively.

\subsection{Statistical Analysis}

All values are presented as mean \pm standard error of the mean (SEM). Permeability data were analyzed using ordinary one-way-ANOVA followed by Tukey's multiple comparisons post hoc test performed using GraphPad Prism (version 9.0.0, GraphPad Software, San Diego, CA, USA). Differences were considered to be statistically significant at $\mathrm{P}<0.05$ ( ${ }^{*} \mathrm{P}<0.05 ;{ }^{* *} \mathrm{P}<0.01 ;{ }^{* * *} \mathrm{P}<0.001 ;{ }^{* * * *} \mathrm{P}<0.0001 ;$ ns $=$ not significant).

Linear regression $\left(\mathrm{R}^{2}\right)$ and Pearson's correlation coefficient $(\mathrm{r})$ analysis was performed using GraphPad Prism (version 9.0.0, GraphPad Software).

\section{Results and Discussion}

The aim of the present study was to establish an in vitro corneal mimetic with a collagen-based self-supporting hydrogel and validate the model as an alternative for the ex vivo rabbit corneas in permeability testing. The permeability of drug molecules, marketed ophthalmic drugs with different physicochemical properties, was tested and compared to permeability values of rabbit corneal tissue.

The hydrogels, serving as the support for epithelial cells and mimicking the stromal layer of the native cornea, contained commercially available porcine collagen type I. It was chemically crosslinked into a self-supporting, transparent material following the procedures developed for synthesis of artificial corneas in cornea transplantation [17]. The crosslinking agent (DMTMM) used in this study allows obtaining a slower crosslinking rate and better hydrogel synthesis control as compared to $\mathrm{N}$-hydroxysuccinimide (NHS)/1-ethyl-3-(3-dimethylaminopropyl)carbodiimide (EDC) crosslinking chemistry [27]. The measured elastic modulus of synthesized collagen hydrogels was $0.36 \pm 0.07$ $\mathrm{MPa}$. It is slightly lower as compared to the collagen hydrogels crosslinked using $\mathrm{NHS} / \mathrm{EDC}$ with elastic modulus $0.60 \pm 0.26 \mathrm{MPa}$ [28]. It is worthwhile to note that the AFM nanoindentation technique used here directly measures the elastic modulus of surface and sub-surface layers of less than $1 \mu \mathrm{m}$ in depth as compared to the bulk elastic moduli measured by other techniques (e.g., oscillatory rheology). Therefore, the AFM nanoindentation technique should yield a value which is arguably closer to the actual elasticity of the sample, as sensed by cells, especially in case of materials having a degree of heterogeneity. Our recent study on hydrogels, manufactured using collagen-like peptide-based formulations, however, did not find a significant divergence between the results yielded by the AFM nanoindentation and oscillatory rheology [29]. We would like to point out that synthesized collagen hydrogels employed in this study had certain physical differences as compared to the native human cornea. The collagen hydrogels used contained $8.5 \pm 0.3 \%$ collagen and $91.5 \pm 0.3 \%$ water in comparison to a human cornea's water content of $78 \%$. However, it is known that other synthetic porcine and recombinant human collagen hydrogels exhibit higher water content $(\sim 90 \%)$ than human cornea [19]. The elastic modulus in human cornea is also higher compared to our collagen hydrogels, 3-13 MPa vs. $0.36 \pm$ $0.07 \mathrm{MPa}$, respectively [19]. Nevertheless, the variation between the DMTMM crosslinked hydrogels samples employed in the current study was minimal, where the standard deviation of the 12 samples was only $0.07 \mathrm{MPa}$. Thus, homogeneous hydrogels were obtained showing negligible structural variation between the samples, a reproducibility that is important for forming consistent cell layer structures and interpretation of results obtained from any drug testing performed on them.

The corneal stroma contains predominantly type I collagen [16] which has been shown to enhance HCE-T cells morphology and adhesion [30]. Therefore, collagen type I 
hydrogels were casted onto a supporting frame (Figure 1) and used to obtain the in vitro corneal mimetic, a multilayered human corneal epithelial layer. The supporting frame was designed to fit between the vertical chambers and to protect the cell layers grown onto the hydrogel during handling and mounting. No signs of the cytotoxicity of the supporting frame were observed during the cell culture, cells were growing normally in close proximity of the supporting frame without any morphological alterations.

A

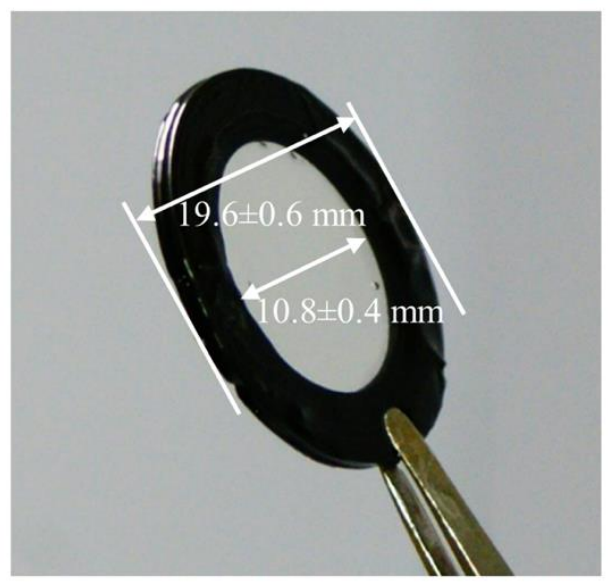

B

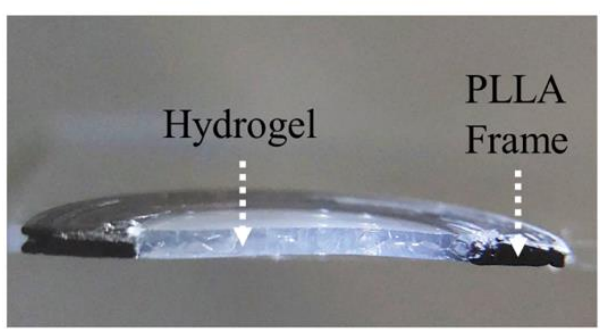

Figure 1. The collagen hydrogel casted onto a plastic circular supporting frame made from poly-Llactic acid (PLLA, black) designed to support the formation of human corneal epithelial multilayer for drug permeability testing in a vertical chamber (A). The cross-section of the transparent hydrogel casted in the supporting frame (B).

The characterization of the developed in vitro human corneal mimetic is shown in Figure 2. Both in vitro corneal models express ZO-1 (Figure 2A-B), which is a responsible for anchoring integral proteins of tight junctions to the actin cytoskeleton [31]. Interestingly, ZO-1 and occludin are expressed in full thickness of in vitro multilayers (Figure 2DE). It is well known that the tight junction barrier is only expressed in the superficial cell layer of the rabbit corneal epithelium (Figure 2F). Indicating that there are significant differences between in vitro and in vivo epithelial phenotypes.

Furthermore, both in vitro corneal models, cells grown in cell culture inserts and the in vitro corneal mimetic with self-supporting hydrogel, exhibited multilayers of epithelial cells (Figure 2G-H). However, the cells grown in cell culture inserts demonstrate much thicker $(100-200 \mu \mathrm{m})$ epithelial cell multilayer than those grown on hydrogel $(50 \mu \mathrm{m})$. The thickness of epithelial cell multilayer in the hydrogel-based corneal mimetic model is corresponding to the thickness of rabbit corneal epithelium (Figure 2I). 

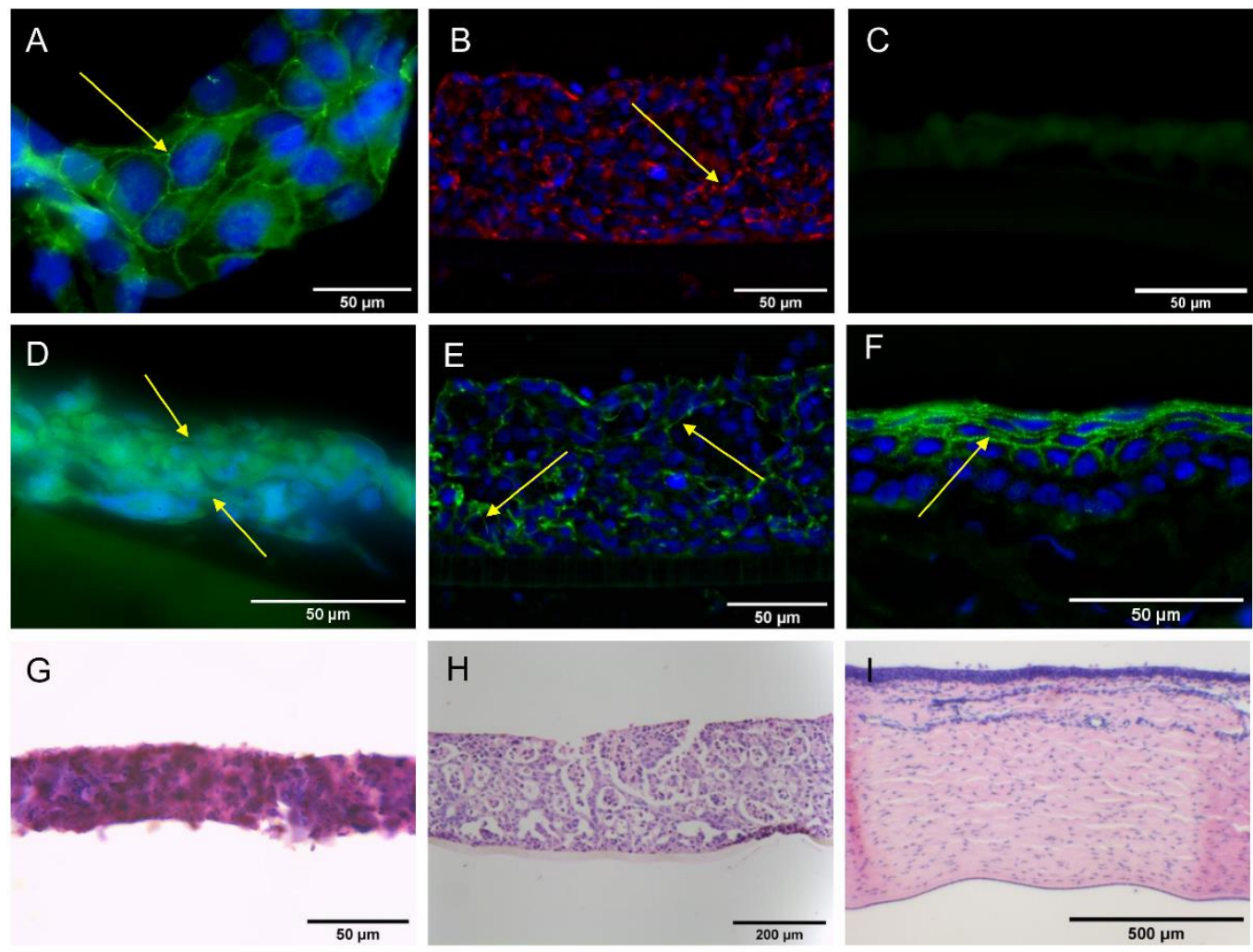

Figure 2. Immunocytochemistry and histology of in vitro corneal models and rabbit cornea. Immunofluorescent staining for zonula occludens-1 (ZO-1) protein expression (green) in HCE-T multilayers grown on the top of the collagen hydrogel (A), human corneal epithelial cells (HCE-T) grown on conventional cell culture insert (red) (B). Negative control of occludin for HCE-T cells grown on top of hydrogel (C). Immunofluorescent staining for occludin (green) in hydrogel-based in vitro corneal mimetic (D), HCE-T cells grown in insert (E) and rabbit cornea (F). Nuclei are stained with DAPI (blue) (A-F). Yellow arrows indicate the tight junctional staining between the cells. Hematoxylin and eosin stained sections of HCE-T multilayers grown on the top of the collagen hydrogel $(\mathbf{G})$, HCE-T cells grown in insert $(\mathbf{H})$ and rabbit cornea $(\mathbf{I})$.

The apparent permeability coefficient ( $\mathrm{Papp}$ ) values for fluorescent reference molecules (6-CF, Rho B, Rho 123, FD4, FD70) and for clinically used topically applied drugs (betaxolol, brinzolamide, dexamethasone, timolol maleate, pilocarpine, chloramphenicol) were determined in the hydrogel-based model and on insert-grown corneal multilayer that lacked stromal equivalent. Tested molecules (Table 1) represent generally used lipophilic and hydrophilic molecules from low and high molecular weight range. The Papp values obtained in this study and the reference values of rabbit corneal permeability from the literature are shown in Table 2.

A tighter paracellular barrier was obtained in the hydrogel-based human corneal model in three weeks of culture, than in the cells grown conventionally in inserts for four weeks (Table 2). In general, the cell layers grown in inserts were more permeable (the range of Papp values was higher). Barrier characteristics are crucially important in in vitro models used for drug permeation studies. While establishing an in vitro model for assessing drug permeability it is important to keep in mind the major layers responsible for limiting the penetration, both the corneal epithelium and stroma. The main barrier for the penetration of hydrophilic molecules is the corneal epithelium [1]. Thus, human cornea is a naturally efficient barrier for different molecules due to its natural characteristics and cellular content. 
Table 2. Papp values across the in vitro hydrogel-based human corneal model, cells grown in cell culture inserts and rabbit cornea.

\begin{tabular}{cccccccc}
\hline Test molecule & $\begin{array}{c}\text { Molecular } \\
\text { weight }\end{array}$ & LogP & $\begin{array}{c}\text { Hydrogel-based } \\
\text { human corneal } \\
\text { model }\end{array}$ & $\begin{array}{c}\text { Cells grown } \\
\text { in inserts }\end{array}$ & $\begin{array}{c}\text { Rabbit } \\
\text { cornea }\end{array}$ & $\begin{array}{c}\text { Rabbit cornea } \\
\text { (values from } \\
\text { literature) }\end{array}$ & Ref. \\
\hline $\begin{array}{c}\text { FITC-dextran } \\
70 \mathrm{kDa}\end{array}$ & 70000 & $-3.29^{*}$ & $0.16 \pm 0.08$ & $0.07 \pm 0.01$ & nd & Impermeable & {$[32]$} \\
\hline $\begin{array}{c}\text { FITC-dextran } \\
4 \mathrm{kDa}\end{array}$ & 4000 & $-3.41^{*}$ & $0.80 \pm 0.52$ & $1.43 \pm 0.25$ & $0.40 \pm 0.09$ & $0.056,0.09$ & {$[10,33]$} \\
\hline Rhodamine 123 & 380.8 & $1.06^{*}$ & $0.96 \pm 0.27$ & $2.22 \pm 0.05$ & $0.42 \pm 0.17$ & 0.15 & {$[34]$} \\
\hline $\begin{array}{c}\text { 6-carboxy- } \\
\text { fluorescein }\end{array}$ & 376.3 & $-3.1^{\$}$ & $0.89 \pm 0.16$ & $2.87 \pm 0.30$ & $1.4 \pm 0.2$ & 0.46 & {$[26]$} \\
\hline Brinzolamide & 383.5 & $-1.8^{*}$ & $1.17 \pm 0.36$ & $8.99 \pm 0.05$ & nd & 0.2 & {$[5]$} \\
\hline Dexamethasone & 392.5 & $1.83^{*}$ & $6.86 \pm 1.13$ & $23.32 \pm 0.20$ & nd & $5,7.7$ & {$[9,35]$} \\
\hline Chloramphenicol & 323.1 & $1.14^{*}$ & $4.75 \pm 0.39$ & $12.79 \pm 0.54$ & nd & 6.8 & {$[35]$} \\
\hline Timolol maleate & 316.4 & $1.1^{*}$ & $29.47 \pm 1.05$ & $20.62 \pm 4.27$ & nd & $11.7,22.5$ & {$[9,35]$} \\
\hline Pilocarpine & 208.3 & $1.1^{*}$ & $16.86 \pm 0.58$ & $39.4 \pm 0.97$ & nd & 17.4 & {$[35]$} \\
\hline Rhodamine B & 479.0 & $2.3^{*}$ & $19.98 \pm 2.47$ & $40.22 \pm 3.02$ & $9.1 \pm 0.9$ & $13.5,18.1$ & {$[10,26]$} \\
\hline Betaxolol & 307.4 & $2.81^{*}$ & $34.56 \pm 3.69$ & $42.00 \pm 3.66$ & nd & 27 & {$[36]$} \\
\hline * Ref. [37]; ${ }^{*}$ Ref. [38]; ${ }^{*}$ Ref. [39]; ${ }^{\$}$ Ref. [26]; nd, not determined. & &
\end{tabular}

Drug's ability to permeate through corneal barriers is linked to its molecular properties. Four properties strongly associated with the drug permeability are known as Lipiski's rule of five: lipophilicity (logarithm of the octanol/water partition coefficient, LogP), number of hydrogen-bond donor groups, number of hydrogen-bond acceptor groups, polar surface area, and molecular weight [40]. Same molecular properties have been shown to influence the in vitro Papp values across different cell models [41]. Sufficient lipophilicity is needed in order to be able to partition into the cell membrane. Lipophilicity depends also on the ionic attractive and repulsive interactions between the drug and the phase into which it is partitioning (e.g. epithelial cell multilayer or collagen hydrogel); lipophilicity $=$ hydrophobicity - polarity + ionic interactions) [42].

Significant differences between the Papp values of hydrogel-based human corneal mimetic and cells grown in inserts were observed (Table 3), FD4 and betaxolol were compounds, which did not show statistically significant differences. Statistically significant differences can be explained by the fact that the corneal mimetic contains stroma-like hydrophilic collagen hydrogel layer which is not present in conventionally insert grown corneal epithelial multilayer. The hydrophilic stroma layer creates a significant barrier for lipophilic molecules such as dexamethasone, betaxolol, and rhodamine B (LogP 1.83, 2.81 and 2.3 (Table 2), respectively). Interestingly, the higher water content of the hydrogel compared to human cornea $(91.5 \%$ vs. $78 \%$ ) did not decrease the Papp value of dexamethasone, betaxolol and Rhodamine B, indicating that the higher water content does not limit the transport through hydrogel-based human corneal mimetic.

In addition, there is a significant difference $(\mathrm{P}<0.0001$, Table 3$)$ between $\mathrm{Papp}$ value of pilocarpine in the hydrogel-based human corneal model $\left(16.86 \pm 0.58 \times 10^{-6} \mathrm{~cm} / \mathrm{s}\right)$ and in the insert-grown corneal multilayer $\left(39.4 \pm 0.97 \times 10^{-6} \mathrm{~cm} / \mathrm{s}\right)$. An un-ionized form of pilocarpine (approx. 50\% at physiological pH [36] has twice as high Papp value across cornea as ionized form of pilocarpine [43]. Thus, higher Papp value might be a result of more favorable partitioning of un-ionized pilocarpine in the insert-grown multilayer. On the contrary, the Papp value of timolol maleate is significantly higher $(\mathrm{P}<0.05$, Table 3$)$ in the hydrogel-based human corneal model $\left(29.47 \pm 1.05 \times 10^{-6} \mathrm{~cm} / \mathrm{s}\right)$ than in the insert-grown corneal multilayer $\left(20.62 \pm 4.27 \times 10^{-6} \mathrm{~cm} / \mathrm{s}\right)$, resulted from the fact that only $1 \%$ of timolol maleate is unionized at physiological $\mathrm{pH}$ [36] and significantly thicker epithelial multilayer in cells cultured on inserts forms a rate-limiting barrier.

Table 3. Statistical comparison of $P$ app values. Differences were considered to be statistically significant at $\mathrm{P}<0.05$. ${ }^{*} \mathrm{P}<0.05$; ${ }^{* *} \mathrm{P}<0.01$; ${ }^{* * *} \mathrm{P}<0.001$; ${ }^{* * * *} \mathrm{P}<0.0001$; ns, not significant. 


\begin{tabular}{|c|c|c|c|}
\hline & $\begin{array}{l}\text { Hydrogel-based human } \\
\text { corneal model }\end{array}$ & $\begin{array}{c}\text { Cells grown in inserts } \\
\text { vs. }\end{array}$ & $\begin{array}{l}\text { Hydrogel-based human } \\
\text { corneal model }\end{array}$ \\
\hline Test molecule & $\begin{array}{c}\text { vs. } \\
\text { Rabbit corneal } \\
\text { permeability }\end{array}$ & $\begin{array}{l}\text { Rabbit corneal } \\
\text { permeability }\end{array}$ & $\begin{array}{l}\text { vs. } \\
\text { Cells grown in inserts }\end{array}$ \\
\hline FITC-dextran 70 kDa & $0.0219 *$ & $0.3789 \mathrm{~ns}$ & $0.0077^{* *}$ \\
\hline FITC-dextran 4 kDa & $0.0081^{* *}$ & $<0.0001^{* * * *}$ & $0.0512 \mathrm{~ns}$ \\
\hline Rhodamine 123 & $0.0044^{* *}$ & $<0.0001^{* * * *}$ & $<0.0001^{* * * *}$ \\
\hline 6-carboxy-fluorescein & $0.9956 \mathrm{~ns}$ & $<0.0001^{* * * *}$ & $<0.0001^{* * * *}$ \\
\hline Brinzolamide & $0.0650 \mathrm{~ns}$ & $<0.0001^{* * * *}$ & $<0.0001^{* * * *}$ \\
\hline Dexamethasone & $0.7991 \mathrm{~ns}$ & $<0.0001^{* * * *}$ & $<0.0001^{* * * *}$ \\
\hline Chloramphenicol & $0.0229^{*}$ & $0.0002^{* * *}$ & $<0.0001^{* * * *}$ \\
\hline Timolol maleate & $0.0084^{* *}$ & $0.4509 \mathrm{~ns}$ & $0.0488^{*}$ \\
\hline Pilocarpine & $0.8231 \mathrm{~ns}$ & $<0.0001^{* * * *}$ & $<0.0001^{* * * *}$ \\
\hline Rhodamine B & $0.0089^{* *}$ & $<0.0001^{* * * *}$ & $<0.0001^{* * * *}$ \\
\hline Betaxolol & $0.2858 \mathrm{~ns}$ & $0.0513 \mathrm{~ns}$ & $0.1389 \mathrm{~ns}$ \\
\hline
\end{tabular}

Interestingly, the high molecular weight reference compound (FD70) exhibited significantly higher $\mathrm{Papp}$ value $(\mathrm{P}<0.01$, Table 3$)$ in hydrogel-based corneal model than in corneal cells grown in inserts. Much thicker multilayer of corneal cells grown in inserts has an impact on Papp value since the tight junctional proteins ( $\mathrm{ZO}-1$ and occludin) were shown to be expressed in full thickness in both in vitro models (Figure 2). FD70 is fully paracellularly transported molecule. Thus, the thicker multilayer with expression of tight junctions in full thickness decrease the Papp value efficiently.

Linear regression analysis showed excellent relationship $\left(R^{2}=0.9297\right)$ between permeability values across in vitro corneal mimetic with hydrogel and rabbit cornea (Figure 3A) with a high Pearson's correlation coefficient $(\mathrm{r}=0.96)$. This indicates that in vitro corneal mimetic with hydrogel layer correlates very well to Papp values obtained by measuring rabbit corneal permeability ex vivo and can be used as a tool for drug permeability testing during drug development. However, the relationship between permeability of current in vitro standard (cells grown on cell culture inserts) and that of rabbit cornea was found to be significantly lower $\left(\mathrm{R}^{2}=0.7991, \mathrm{r}=0.89\right)$ (Figure 3B).

A

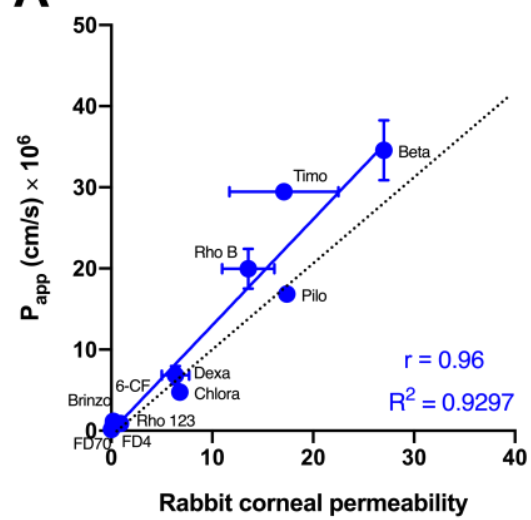

B

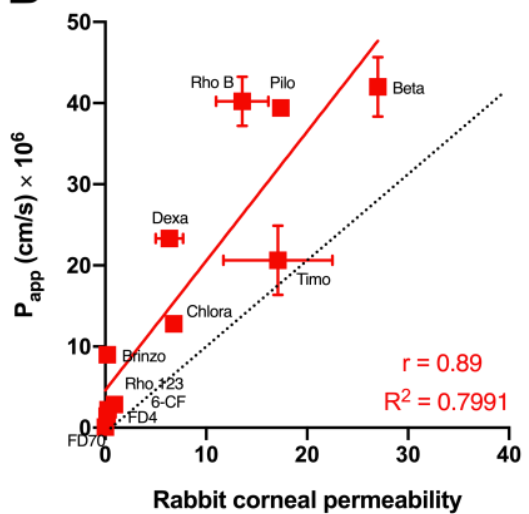

Figure 3. Linear regression between the apparent permeability coefficient, $P$ app $(\mathrm{cm} / \mathrm{s}) \times 10^{6}$, of ophthalmic drugs and reference molecules across in vitro hydrogel-based corneal mimetic (A), the human corneal cells grown conventionally in cell culture inserts (B) and rabbit corneal permeability values. Data are mean \pm SEM. Pearson's correlation coefficients (r) for the in vitro corneal mimetic with hydrogel and the cells grown in cell culture inserts were $r=0.96$ and $r=0.89$, respectively. The dotted lines in the figures represents the perfect positive correlation $(\mathrm{r}=1)$. The goodness of the correlation fit ( $\mathrm{R}^{2}$ value) describes how well the corneal mimetic in vitro model agrees the corresponding rabbit corneal permeability values.

This study further confirms that that human corneal in vitro mimetic requires hydrophilic stroma-like structure in order to be able reliably predict the permeability 
coefficients. Currently, there are available established corneal in vitro models having hydrophilic stroma-like structure formed using a rat tail collagen combined with keratocytes, stromal stem cells and neuronal cells [8-10] Together these studies verify the use of immortalized HCE-T cells suitability for permeability testing, although the HCE-T cells may not fully represent the in vivo cornea architecture or tight junctional localization (Figure 2), multidrug resistance-associated protein expressions [44] or cornea-specific differentiation markers [45].

Previously, human corneal cells have been grown on NHS/EDC crosslinked collagen hydrogels and the diffusion permeability of high molecular weight $(66 \mathrm{kDa})$ reference molecule was in the same range as in the present study [19]. In the same study reliable stability and integration of the hydrogel implant was demonstrated in rabbit and porcine corneas over the period of 6 months after the implantation [19]. However, no previous data on permeability of different molecules across human corneal cells grown on collagen hydrogels crosslinked with NHS/EDC or DMTMM is available. Therefore, the present study provides the first systematic comparison of ophthalmic drug permeability between an in vitro hydrogel-based human corneal mimetic, human corneal epithelial cells grown conventionally in inserts and the ex vivo gold standard, rabbit corneal permeability.

\section{Conclusions}

In this study, the successful development and testing of a unique in vitro human corneal model, composed of two major layers of cornea, containing both a stromal equivalent as well as epithelial cells, is described. The model is based on the human corneal epithelial cells multilayered on a type I collagen-based hydrogel which exhibits permeability that correlates very well to values obtained by measuring rabbit corneal permeability ex vivo. Therefore, we conclude that this in vitro human cornea mimetic can be used for drug permeability tests, whilst avoiding ethical issues and the higher expenses incurred.

Author Contributions: Conceptualization, V.C., R.V., G.K. and J.J.H.; methodology, A.Z. and V.C.; validation, A.Z., V.C. and J.J.H.; formal Analysis, A.Z., J.J.H.; investigation, A.Z., V.C., T.J., R.E. and A.U.; resources, R.V. and G.K.; writing - original draft preparation, A.Z., V.C., R.A., and J.J.H.; writing - review \& editing, V.C., R.A., G.K. and J.J.H.; visualization, A.Z., V.C., R.A. and J.J.H.; supervision, V.C., R.V., G.K. and J.J.H.; project administration, R.V., G.K. and J.J.H.; funding acquisition, R.V., G.K. and J.J.H. All authors have read and agreed to the published version of the manuscript.

Funding: This work was supported by the Eurostars E! 9777 (The Finnish Funding Agency for Technology and Innovation, formerly Tekes, Finland, and Agency for Science, innovation and Technology, Lithuania).

Acknowledgments: The authors would like to thank Dr. Olga Vergun (Experimentica Ltd.) for technical assistance.

Conflicts of Interest: Agnẻ Žiniauskaitė, M.Sc., an employee of Experimentica Ltd. Dr. Vytautas Cépla, an employee of UAB Ferentis. Tadas Jelinskas, M.Sc., an employee of UAB Ferentis. Romuald Eimont, M.Sc., an employee of UAB Ferentis. Artūras Ulčinas, none. Dr. Ruta Aldonytė, an employee of UAB Ferentis. Dr. Ramūnas Valiokas, is a majority shareholder and the chief executive officer of UAB Ferentis, head of Department of Nanoengineering, Center for Physical Sciences and Technology, Vilnius, Lithuania. Dr. Giedrius Kalesnykas has equity ownership in, serves on the executive leadership committee of, as well as being the chief executive officer of Experimentica Ltd. Dr. Jenni J. Hakkarainen has equity ownership in, serves on the executive leadership committee of, and is the chief operating officer of Experimentica Ltd.

The funders had no role in the design of the study; in the collection, analyses, or interpretation of data; in the writing of the manuscript, or in the decision to publish the results.

References

1. Cholkar, K.; Patel, S.P.; Vadlapudi, A.D.; Mitra, A.K. Novel strategies for anterior segment ocular drug delivery. J. Ocul. 
Pharmacol. Ther. 2013, 29, 106-123, doi:10.1089/jop.2012.0200.

2. Mantelli, F.; Mauris, J.; Argüeso, P. The ocular surface epithelial barrier and other mechanisms of mucosal protection: from allergy to infectious diseases. Curr. Opin. Allergy Clin. Immunol. 2013, 13.

3. Directive of the European parliament and of the council on the protection of animals used for scientific purposes. Directive 2010/63/EU. 2010. https://eur-lex.europa.eu/eli/dir/2010/63/oj;

4. Kouchak, M.; Bahmandar, R.; Bavarsad, N.; Farrahi, F. Ocular Dorzolamide Nanoliposomes for Prolonged IOP Reduction: in-vitroand in-vivo Evaluation in Rabbits. Iran. J. Pharm. Res. IJPR 2016, 15, 205-212.

5. Palma, S.D.; Tartara, L.I.; Quinteros, D.; Allemandi, D.A.; Longhi, M.R.; Granero, G.E. An efficient ternary complex of acetazolamide with HP-ss-CD and TEA for topical ocular administration. J. Control. Release 2009, 138, 24-31, doi:10.1016/j.jconrel.2009.04.035.

6. Sharif Makhmalzadeh, B.; Salimi, A.; Niroomand, A. Loratadine-Loaded Thermoresponsive Hydrogel: Characterization and Ex-vivo Rabbit Cornea Permeability Studies. Iran. J. Pharm. Res. IJPR 2018, 17, 460-469.

7. Kaluzhny, Y.; Kinuthia, M.W.; Truong, T.; Lapointe, A.M.; Hayden, P.; Klausner, M. New Human Organotypic Corneal Tissue Model for Ophthalmic Drug Delivery Studies. Invest. Ophthalmol. Vis. Sci. 2018, 59, 2880-2898, doi:10.1167/iovs.1823944.

8. Wang, S.; Ghezzi, C.E.; Gomes, R.; Pollard, R.E.; Funderburgh, J.L.; Kaplan, D.L. In vitro 3D corneal tissue model with epithelium, stroma, and innervation. Biomaterials 2017, 112, 1-9, doi:10.1016/j.biomaterials.2016.09.030.

9. Hahne, M.; Zorn-Kruppa, M.; Guzman, G.; Brandner, J.M.; Haltner-Ukomado, E.; Wätzig, H.; Reichl, S. Prevalidation of a human cornea construct as an alternative to animal corneas for in vitro drug absorption studies. J. Pharm. Sci. 2012, 101, 29762988, doi:10.1002/jps.23190.

10. Hahne, M.; Reichl, S. Development of a serum-free human cornea construct for in vitro drug absorption studies: the influence of varying cultivation parameters on barrier characteristics. Int. J. Pharm. 2011, 416, 268-279, doi:10.1016/j.ijpharm.2011.07.004

11. Yamaguchi, H.; Takezawa, T. Fabrication of a Corneal Model Composed of Corneal Epithelial and Endothelial Cells via a Collagen Vitrigel Membrane Functioned as an Acellular Stroma and Its Application to the Corneal Permeability Test of Chemicals. Drug Metab. Dispos. 2018, 46, 1684-1691, doi:10.1124/dmd.118.080820.

12. Chang, J.-E.; Basu, S.K.; Lee, V.H.L. Air-Interface Condition Promotes the Formation of Tight Corneal Epithelial Cell Layers for Drug Transport Studies. Pharm. Res. 2000, 17, 670-676, doi:10.1023/A:1007569929765.

13. Scholz, M.; Chang Lin, J.-E.; Lee, V.H.L.; Keipert, S. Pilocarpine Permeability across Ocular Tissues and Cell Cultures: Influence of Formulation Parameters. J. Ocul. Pharmacol. Ther. 2002, 18, 455-468.

14. Reichl, S.; Kölln, C.; Hahne, M.; Verstraelen, J. In vitro cell culture models to study the corneal drug absorption. Expert Opin. Drug Metab. Toxicol. 2011, 7, 559-578, doi:10.1517/17425255.2011.562195.

15. Almubrad, T.; Akhtar, S. Structure of corneal layers, collagen fibrils, and proteoglycans of tree shrew cornea. Mol. Vis. 2011, 17, 2283-2291.

16. Ihanamäki, T.; Pelliniemi, L.J.; Vuorio, E. Collagens and collagen-related matrix components in the human and mouse eye. Prog. Retin. Eye Res. 2004, 23, 403-434, doi:https://doi.org/10.1016/j.preteyeres.2004.04.002.

17. Korjamo, T.; Heikkinen, A.T.; Waltari, P.; Mönkkönen, J. The Asymmetry of the Unstirred Water Layer in Permeability Experiments. Pharm. Res. 2008, 25, 1714, doi:10.1007/s11095-008-9573-8.

18. Jansen, K.A.; Donato, D.M.; Balcioglu, H.E.; Schmidt, T.; Danen, E.H.J.; Koenderink, G.H. A guide to mechanobiology: Where biology and physics meet. Biochim. Biophys. Acta - Mol. Cell Res. 2015, 1853, 3043-3052, doi:https://doi.org/10.1016/j.bbamcr.2015.05.007.

19. Liu, Y.; Gan, L.; Carlsson, D.J.; Fagerholm, P.; Lagali, N.; Watsky, M.A.; Munger, R.; Hodge, W.G.; Priest, D.; Griffith, M. A Simple, Cross-linked Collagen Tissue Substitute for Corneal Implantation. Invest. Ophthalmol. Vis. Sci. 2006, 47, 1869-1875, doi:10.1167/iovs.05-1339.

20. Haagdorens, M.; Cẻpla, V.; Melsbach, E.; Koivusalo, L.; Skottman, H.; Griffith, M.; Valiokas, R.; Zakaria, N.; Pintelon, I.; 
Tassignon, M.-J. In Vitro Cultivation of Limbal Epithelial Stem Cells on Surface-Modified Crosslinked Collagen Scaffolds. Stem Cells Int. 2019, 2019, 7867613, doi:10.1155/2019/7867613.

21. Domke, J.; Radmacher, M. Measuring the Elastic Properties of Thin Polymer Films with the Atomic Force Microscope. Langmuir 1998, 14, 3320-3325, doi:10.1021/la9713006.

22. Sokolov, I.; Dokukin, M.E.; Guz, N. V Method for quantitative measurements of the elastic modulus of biological cells in AFM indentation experiments. Methods 2013, 60, 202-213, doi:https://doi.org/10.1016/j.ymeth.2013.03.037.

23. Hutter, J.L.; Bechhoefer, J. Calibration of atomic-force microscope tips. Rev. Sci. Instrum. 1993, 64, 1868-1873, doi:10.1063/1.1143970.

24. Araki-Sasaki, K.; Ohashi, Y.; Sasabe, T.; Hayashi, K.; Watanabe, H.; Tano, Y.; Handa, H. An SV40-immortalized human corneal epithelial cell line and its characterization. Invest. Ophthalmol. Vis. Sci. 1995, 36, 614-621.

25. Hakkarainen, J.J.; Reinisalo, M.; Ragauskas, S.; Seppänen, A.; Kaja, S.; Kalesnykas, G. Acute cytotoxic effects of marketed ophthalmic formulations on human corneal epithelial cells. Int. J. Pharm. 2016, 511, 73-78, doi:https://doi.org/10.1016/j.ijpharm.2016.06.135.

26. Toropainen, E.; Ranta, V.P.; Talvitte, A.; Suhonen, P.; Urtti, A. Culture model of human corneal epithelium for prediction of ocular drug absorption. Investig. Ophthalmol. Vis. Sci. 2001, 42, 2942-2948.

27. D'Este, M.; Eglin, D.; Alini, M. A systematic analysis of DMTMM vs EDC/NHS for ligation of amines to Hyaluronan in water. Carbohydr. Polym. 2014, 108, 239-246, doi:https://doi.org/10.1016/j.carbpol.2014.02.070.

28. Liu, W.; Deng, C.; McLaughlin, C.R.; Fagerholm, P.; Lagali, N.S.; Heyne, B.; Scaiano, J.C.; Watsky, M.A.; Kato, Y.; Munger, R.; et al. Collagen-phosphorylcholine interpenetrating network hydrogels as corneal substitutes. Biomaterials 2009, 30, 15511559, doi:https://doi.org/10.1016/j.biomaterials.2008.11.022.

29. Balion, Z.; Cejpla, V.; Svirskiene, N.; Svirskis, G.; Druceikaitè, K.; Inokaitis, H.; Rusteikaitè, J.; Masilionis, I.; Stankevičienė, G.; Jelinskas, T.; et al. Cerebellar Cells Self-Assemble into Functional Organoids on Synthetic, Chemically Crosslinked ECMMimicking Peptide Hydrogels. Biomolecules 2020, 10, doi:10.3390/biom10050754.

30. Kimura, K.; Kawano, S.; Mori, T.; Inoue, J.; Hadachi, H.; Saito, T.; Nishida, T. Quantitative analysis of the effects of extracellular matrix proteins on membrane dynamics associated with corneal epithelial cell motility. Invest. Ophthalmol. Vis. Sci. 2010, 51, 4492-4499, doi:10.1167/iovs.09-4380.

31. Wu, P.; Gong, H.; Richman, R.; Freddo, T.F. Localization of occludin, ZO-1, and pan-cadherin in rabbit ciliary epithelium and iris vascular endothelium. Histochem. Cell Biol. 2000, 114, 303-310, doi:10.1007/s004180000195.

32. Huang, A.J.; Tseng, S.C.; Kenyon, K.R. Paracellular permeability of corneal and conjunctival epithelia. Invest. Ophthalmol. Vis. Sci. 1989, 30, 684-689.

33. Sasaki, H.; Yamamura, K.; Tei, C.; Nishida, K.; Nakamura, J. Ocular Permeability of FITC-Dextran with Absorption Promoter for Ocular Delivery of Peptide Drug. J. Drug Target. 1995, 3, 129-135, doi:10.3109/10611869509059212.

34. Becker, U.; Ehrhardt, C.; Daum, N.; Baldes, C.; Schaefer, U.F.; Ruprecht, K.W.; Kim, K.J.; Lehr, C.M. Expression of ABCtransporters in human corneal tissue and the transformed cell line, HCE-T. J. Ocul. Pharmacol. Ther. 2007, 23, 172-181, doi:10.1089/jop.2006.0095.

35. Schoenwald, R.D. Ocular drug delivery. Pharmacokinetic considerations. Clin. Pharmacokinet. 1990, 18(4), $255-269$.

36. Prausnitz, M.R.; Noonan, J.S. Permeability of cornea, sclera, and conjunctiva: A literature analysis for drug delivery to the eye. J. Pharm. Sci. 1998, 87, 1479-1488, doi:10.1021/js9802594.

37. National Center for Biotechnology Information (2021). PubChem Compound Summary. Retrieved February 13, 2021 from https://pubchem.ncbi.nlm.nih.gov/.

38. Tomita, M.; Menconi, M.J.; Delude, R.L.; Fink, M.P. Polarized transport of hydrophilic compounds across rat colonic mucosa from serosa to mucosa is temperature dependent. Gastroenterology 2000, 118, 535-543, doi:10.1016/s0016-5085(00)70259-9.

39. Duvvuri, M.; Gong, Y.; Chatterji, D.; Krise, J.P. Weak base permeability characteristics influence the intracellular sequestration site in the multidrug-resistant human leukemic cell line HL-60. J. Biol. Chem. 2004, 279, 32367-32372, 
doi:10.1074/jbc.M400735200.

40. Lipinski, C.A.; Lombardo, F.; Dominy, B.W.; Feeney, P.J. Experimental and computational approaches to estimate solubility and permeability in drug discovery and development settings. Adv. Drug Deliv. Rev. 2001, 46, 3-26, doi:10.1016/s0169409x(00)00129-0.

41. Hakkarainen, J.J.; Pajander, J.; Laitinen, R.; Suhonen, M.; Forsberg, M.M. Similar molecular descriptors determine the in vitro drug permeability in endothelial and epithelial cells. Int. J. Pharm. 2012, 436, 426-443, doi:10.1016/j.ijpharm.2012.06.049.

42. Liu, X.; Testa, B.; Fahr, A. Lipophilicity and its relationship with passive drug permeation. Pharm. Res. 2011, 28, 962-977, doi:10.1007/s11095-010-0303-7.

43. Mitra, A.K.; Mikkelson, T.J. Mechanism of transcorneal permeation of pilocarpine. J. Pharm. Sci. 1988, 77, 771-775, doi:10.1002/jps.2600770911.

44. Verstraelen, J.; Reichl, S. Multidrug resistance-associated protein (MRP1, 2, 4 and 5) expression in human corneal cell culture models and animal corneal tissue. Mol. Pharm. 2014, 11, 2160-2171, doi:10.1021/mp400625z.

45. Rubelowski, A.-K.; Latta, L.; Katiyar, P.; Stachon, T.; Käsmann-Kellner, B.; Seitz, B.; Szentmáry, N. HCE-T cell line lacks cornea-specific differentiation markers compared to primary limbal epithelial cells and differentiated corneal epithelium. Graefe's Arch. Clin. Exp. Ophthalmol. 2020, 258, 565-575, doi:10.1007/s00417-019-04563-0. 